FedUni ResearchOnline

http://researchonline.federation.edu.au

This is the author's preprint version of the following publication:

Zhou, X., Gao, D., Yang, C. (2016) Global solutions to a class of

CEC benchmark constrained optimization problems. Optimization

Letters, 10(3), 457-472.

The version displayed here may differ from the final published version.

The final publication is available at Springer via:

http://doi.org/10.1007/s11590-014-0784-0

Copyright @ 2014, Springer-Verlag Berlin Heidelberg. 


\title{
Global solutions to a class of CEC benchmark constrained optimization problems
}

\author{
Xiaojun Zhou • David Yang Gao • \\ Chunhua Yang
}

Received: date / Accepted: date

\begin{abstract}
This paper aims to solve a class of CEC benchmark constrained optimization problems that have been widely studied by nature-inspired optimization algorithms. Global optimality condition based on canonical duality theory is derived. Integrating the dual solutions with the KKT conditions, we are able to obtain the approximate solutions or global solutions easily.
\end{abstract}

Keywords Global optimization · Constrained optimization · Canonical duality theory · CEC benchmark

\section{Introduction}

Nature-inspired optimization algorithms, such as genetic algorithm (GA), evolution strategy (ES), particle swarm optimization (PSO) and differential evolution (DE), have received considerable attention in recent decades due to their strong adaptability and easy implementation. Strictly speaking, these algorithms are unconstrained optimization procedures, and therefore it is necessary to find techniques to deal with the constraints when solving constrained optimization problems. The most common approach to handle constraints is

Xiaojun Zhou

School of Science, Information Technology and Engineering, University of Ballarat, Victoria 3353, Australia.

School of Information Science and Engineering, Central South University, Changsha 410083, China.

David Yang Gao

School of Science, Information Technology and Engineering, University of Ballarat, Victoria 3353, Australia.

Chunhua Yang

School of Information Science and Engineering, Central South University, Changsha 410083, China. 
the penalty function method. The idea of this approach is to transform a constrained optimization problem into an unconstrained one by adding a certain term to the objective function based on the amount of constraint violation. Then, some special representations and operators are designed to preserve the feasibility of solutions at all times or to repair a solution when it is infeasible. Multiobjective optimization techniques are also applied to manage constraints. The main idea is to rewrite the single objective optimization problem as a multiobjective optimization problem in which the constraints in original problem are treated as additional objectives 3,10.

By introducing a Lagrangian multiplier vector to relax the constraints, the classical Lagrangian is a saddle function if the objective function and all of the constraints are convex. Under certain constraint qualifications, the well-known strong min-max duality relation holds, and in this case, the problem can be easily solved by well-developed convex programming techniques. However, due to nonconvexity of either objective function or the constraints, the Lagrangian is no longer a saddle function and only the weak duality relation holds, leading to the duality gap in global optimization [1,2,6. In order to bridge the gap inherent in the classical Lagrange duality theory, the canonical duality theory has been developed recently. Its core is to transform a nonconvex primal minimization problem to the concave canonical dual maximization problem over a convex space without duality gap by a canonical dual transformation [7].

As shown by the global optimality condition contained in the canonical duality theory, if the dual solution is in the positive definite domain, it is easy to get the corresponding global solution to the primal problem. However, if the condition is not satisfied, some strategies are necessary to recover the global solution [5, 8, 12. In this paper, we focus on solving a class of CEC (Congress on Evolutionary Computation) benchmark constrained optimization problems that have been widely studied by nature-inspired algorithms. By integrating the canonical duality theory with the KKT conditions, we are able to obtain the approximate solutions or global solutions easily.

\section{The canonical duality theory}

In this paper, we focus on the following quadratic optimization problem with quadratic and box constraints (primal problem):

$$
\begin{aligned}
(\mathcal{P}): \min & \left\{P(\mathbf{x})=\frac{1}{2} \mathbf{x}^{T} A \mathbf{x}-\mathbf{a}^{T} \mathbf{x}-a: \mathbf{x} \in \mathbb{R}^{n}\right\} \\
\text { s.t. } & \mathbf{g}(\mathbf{x})=\left\{g_{j}(\mathbf{x})\right\}=\left\{\frac{1}{2} \mathbf{x}^{T} B_{j} \mathbf{x}-\mathbf{b}_{j}^{T} \mathbf{x}-b_{j}\right\} \leq \mathbf{0}, j=1, \cdots, m, \\
& c_{i} \leq x_{i} \leq d_{i}, i=1, \cdots, n,
\end{aligned}
$$

where, $\mathbf{x}=\left(x_{1}, \cdots, x_{n}\right), A=A^{T}, B_{j}=B_{j}^{T} \in \mathbb{R}^{n \times n}$ are symmetric matrices, $\mathbf{a}, \mathbf{b}_{j} \in \mathbb{R}^{n}$ are given vectors, $a, b_{j}, c_{i}, d_{i}$ are constant.

Let $E_{i} \in \mathbb{R}^{n \times n}, \mathbf{e}_{i} \in \mathbb{R}^{n}$ be a diagonal matrix and a unit vector, with all zeros except a one in the position $(i, i)$ and $(i)$, respectively, $B_{k}=2 E_{k}$, 
$\mathbf{b}_{k}=\left(c_{k}+d_{k}\right) \mathbf{e}_{k}, b_{k}=c_{k} d_{k}, k=m+1, \cdots, m+n$, then constraints in $(\mathcal{P})$ can be uniformly rewritten to

$$
\mathbf{g}(\mathbf{x})=\left\{g_{k}(\mathbf{x})\right\}=\left\{\frac{1}{2} \mathbf{x}^{T} B_{k} \mathbf{x}-\mathbf{b}_{k}^{T} \mathbf{x}-b_{k}\right\} \leq \mathbf{0}, k=1, \cdots, m+n .
$$

Firstly, we introduce an indicator function:

$$
W(\boldsymbol{\epsilon})=\left\{\begin{aligned}
0 & \text { if } \boldsymbol{\epsilon} \leq \mathbf{0} \\
+\infty & \text { otherwise }
\end{aligned}\right.
$$

where, $\boldsymbol{\epsilon}=\left(\epsilon_{1}, \cdots, \epsilon_{m+n}\right)$, and let $U(\mathbf{x})=-f(\mathbf{x})=-\frac{1}{2} \mathbf{x}^{T} A \mathbf{x}+\mathbf{a}^{T} \mathbf{x}+a$, then the primal problem $(\mathcal{P})$ can be written in the following form:

$$
\min \left\{\Pi_{0}(\mathbf{x})=W(\mathbf{g}(\mathbf{x}))-U(\mathbf{x}): \mathbf{x} \in \mathbb{R}^{n}\right\} .
$$

Secondly, we introduce a nonlinear operator

$$
\boldsymbol{\xi}=\left(\xi_{1}, \cdots, \xi_{m+n}\right)=\left\{\xi_{k}\right\}=\Lambda(\mathbf{x})=\left\{\frac{1}{2} \mathbf{x}^{T} B_{k} \mathbf{x}-\mathbf{b}_{k}^{T} \mathbf{x}-b_{k}\right\}
$$

so that $\mathbf{g}(\mathbf{x})$ can be recast by $V(\boldsymbol{\xi})=\boldsymbol{\xi}=\mathbf{g}(\mathbf{x})$, and then the primal problem $(\mathcal{P})$ can be reformed in the canonical form:

$$
\min \left\{\Pi(\mathbf{x})=W(V(\Lambda(\mathbf{x})))-U(\mathbf{x}): \mathbf{x} \in \mathbb{R}^{n}\right\} .
$$

By the Fenchel transformation, the conjugate function $W^{\sharp}(\boldsymbol{\sigma})$ of $W(\boldsymbol{\epsilon})$ can be defined by

$$
W^{\sharp}(\boldsymbol{\sigma})=\sup _{\boldsymbol{\epsilon}}\left\{\boldsymbol{\epsilon}^{T} \boldsymbol{\sigma}-W(\boldsymbol{\epsilon})\right\}=\left\{\begin{aligned}
0 & \text { if } \boldsymbol{\sigma} \geq 0 \\
+\infty & \text { otherwise }
\end{aligned}\right.
$$

which is convex and lower semi-continuous.

According to the relations $\boldsymbol{\sigma} \in \partial W(\boldsymbol{\epsilon}) \Leftrightarrow \boldsymbol{\epsilon} \in \partial W^{\sharp}(\boldsymbol{\sigma}) \Leftrightarrow W(\boldsymbol{\epsilon})+W^{\sharp}(\boldsymbol{\sigma})=$ $\boldsymbol{\epsilon}^{T} \boldsymbol{\sigma}$ from convex analysis, we can replace $W(\mathbf{g}(\mathbf{x}))$ by $\mathbf{g}^{T}(\mathbf{x}) \boldsymbol{\sigma}-W^{\sharp}(\boldsymbol{\sigma})$, and then we can get the extended Lagrangian

$$
\Xi_{0}(\mathbf{x}, \boldsymbol{\sigma})=\mathbf{g}^{T}(\mathbf{x}) \boldsymbol{\sigma}-W^{\sharp}(\boldsymbol{\sigma})-U(\mathbf{x}) .
$$

Next, we introduce the invertible duality mapping

$$
\varsigma=\left(\varsigma_{1}, \cdots, \varsigma_{m+n}\right)=\left\{\varsigma_{k}\right\}=\nabla V(\boldsymbol{\xi})=I .
$$

Defining the Legendre conjugate $V^{*}(\boldsymbol{\varsigma})=\operatorname{sta}\left\{\boldsymbol{\xi}^{T} \boldsymbol{\varsigma}-V(\boldsymbol{\xi})\right\}$, and using the equivalent relations $\boldsymbol{\varsigma}=\nabla V(\boldsymbol{\xi}) \Leftrightarrow \boldsymbol{\xi}=\nabla V^{*}(\boldsymbol{\varsigma}) \Leftrightarrow \boldsymbol{\xi}^{T} \boldsymbol{\varsigma}=V(\boldsymbol{\xi})+V^{*}(\boldsymbol{\varsigma})$, we can replace $\mathbf{g}(\mathbf{x})$ by $\Lambda^{T}(\mathbf{x}) \boldsymbol{\varsigma}-V^{*}(\boldsymbol{\varsigma})$, so we obtain the generalized complementary function

$$
\Xi(\mathbf{x}, \boldsymbol{\sigma})=\frac{1}{2} \mathbf{x}^{T} G(\boldsymbol{\sigma}) \mathbf{x}-\mathbf{x}^{T} F(\boldsymbol{\sigma})-\boldsymbol{\sigma}^{T} \mathbf{d}-a,
$$


where,

$$
G(\boldsymbol{\sigma})=A+\sum_{k=1}^{m+n} \sigma_{k} B_{k}, F(\boldsymbol{\sigma})=\mathbf{a}+\sum_{k=1}^{m+n} \sigma_{k} \mathbf{b}_{k}
$$

and

$$
\mathbf{d}=\left(b_{1}, \cdots, b_{m+n}\right)^{T}, \boldsymbol{\sigma}=\left(\sigma_{1}, \cdots, \sigma_{m+n}\right) \in \mathbb{R}_{+}^{m+n}=\left\{\boldsymbol{\sigma} \in \mathbb{R}^{m+n} \mid \boldsymbol{\sigma} \geq 0\right\} .
$$

By using the generalized complementary function, the canonical dual function $P^{d}(\boldsymbol{\sigma})$ can be formulated as

$$
P^{d}(\boldsymbol{\sigma})=\operatorname{sta}_{\mathbf{x}}\{\Xi(\mathbf{x}, \boldsymbol{\sigma})\} .
$$

Solving the critical points of $\Xi(\mathbf{x}, \boldsymbol{\sigma})$, we can get the canonical equilibrium equation

$$
G(\boldsymbol{\sigma}) \mathbf{x}=F(\boldsymbol{\sigma}) .
$$

For any given $\boldsymbol{\sigma}$, if $F(\boldsymbol{\sigma})$ is in the column space of $G(\boldsymbol{\sigma})$, denoted by $\mathcal{C}_{o l}(G(\boldsymbol{\sigma}))$, i.e., a linear space spanned by the columns of $G(\boldsymbol{\sigma})$, the solution of the canonical equilibrium equation can be well defined by

$$
\mathbf{x}=G^{\dagger}(\boldsymbol{\sigma}) F(\boldsymbol{\sigma})
$$

where, $G^{\dagger}(\boldsymbol{\sigma})$ denotes the Moore-Penrose generalized inverse of $G(\boldsymbol{\sigma})$.

Then, the canonical dual function can be written explicitly as follows

$$
P^{d}(\boldsymbol{\sigma})=-\frac{1}{2} F^{T}(\boldsymbol{\sigma}) G^{\dagger}(\boldsymbol{\sigma}) F(\boldsymbol{\sigma})-\boldsymbol{\sigma}^{T} \mathbf{d}-a .
$$

Finally, the canonical dual problem can be posed as follows:

$$
\left(\mathcal{P}^{d}\right): \max \left\{P^{d}(\boldsymbol{\sigma}): \boldsymbol{\sigma} \in \mathcal{S}_{a}^{+}\right\}
$$

where, the dual feasible space is defined by $\mathcal{S}_{a}^{+}=\left\{\boldsymbol{\sigma} \in \mathbb{R}_{+}^{m+n} \mid G(\boldsymbol{\sigma}) \succeq 0\right\}$.

Theorem 1 (Global Optimality Condition). Suppose $\overline{\boldsymbol{\sigma}}$ is a KKT point of $\left(\mathcal{P}^{d}\right)$. If $G(\overline{\boldsymbol{\sigma}}) \succ 0$, then $\overline{\mathbf{x}}=G^{-1}(\overline{\boldsymbol{\sigma}}) F(\overline{\boldsymbol{\sigma}})$ is the global minimizer of $(\mathcal{P})$. If $\operatorname{det}(G(\overline{\boldsymbol{\sigma}}))=0$, the global minimizer $\overline{\mathbf{x}}$ of $(\mathcal{P})$ is contained in the canonical equilibrium equation $G(\overline{\boldsymbol{\sigma}}) \overline{\mathbf{x}}=F(\overline{\boldsymbol{\sigma}})$.

Proof. By introducing Lagrange multiplier $\boldsymbol{\epsilon} \in \mathbb{R}_{-}^{m+n}$ (where $\mathbb{R}_{-}^{m+n}$ is the nonpositive orthant of $\mathbb{R}^{m+n}$ ) associated with $\boldsymbol{\sigma} \geq 0$, the Lagrangian $L(\boldsymbol{\epsilon}, \boldsymbol{\sigma})$ is given by

$$
L(\boldsymbol{\epsilon}, \boldsymbol{\sigma})=-\frac{1}{2} F^{T}(\boldsymbol{\sigma}) G^{\dagger}(\boldsymbol{\sigma}) F(\boldsymbol{\sigma})-\boldsymbol{\sigma}^{T} \mathbf{d}-a-\boldsymbol{\epsilon}^{T} \boldsymbol{\sigma} .
$$

It is easy to prove that the criticality conditions $\nabla_{\boldsymbol{\sigma}} L(\boldsymbol{\epsilon}, \boldsymbol{\sigma})=0$ lead to

$$
\boldsymbol{\epsilon}=\left(\begin{array}{c}
\epsilon_{1} \\
\cdots \\
\epsilon_{m+n}
\end{array}\right)=\left(\begin{array}{c}
\frac{1}{2} \overline{\mathbf{x}}^{T} B_{1} \overline{\mathbf{x}}-\mathbf{b}_{1}^{T} \overline{\mathbf{x}}-b_{1} \\
\cdots \\
\frac{1}{2} \overline{\mathbf{x}}^{T} B_{m+n} \overline{\mathbf{x}}-\mathbf{b}_{m+n}^{T} \overline{\mathbf{x}}-b_{m+n}
\end{array}\right)
$$


and the accompanying KKT conditions include

$$
0 \leq \bar{\sigma}_{k} \perp \frac{1}{2} \overline{\mathbf{x}}^{T} B_{k} \overline{\mathbf{x}}-\mathbf{b}_{k}^{T} \overline{\mathbf{x}}-b_{k} \leq 0, k=1, \cdots, m+n .
$$

From the complementary slackness, we can see that the $\overline{\mathbf{x}}$ satisfies the constraints in $(\mathcal{P})$. Furthermore, since $\overline{\boldsymbol{\sigma}} \geq 0$ for any $\mathbf{g}(\mathbf{x}) \leq 0$, we have

$$
\begin{aligned}
P(\mathbf{x}) & \geq P(\mathbf{x})+\overline{\boldsymbol{\sigma}}^{T} \mathbf{g}(\mathbf{x}) \\
& =\frac{1}{2} \mathbf{x}^{T} A \mathbf{x}-\mathbf{a}^{T} \mathbf{x}-a+\sum_{k=1}^{m+n}\left(\frac{1}{2} \mathbf{x}^{T} \bar{\sigma}_{k} B_{k} \mathbf{x}-\bar{\sigma}_{k} \mathbf{b}_{k}^{T} \mathbf{x}-\bar{\sigma}_{k} b_{k}\right) \\
& =\frac{1}{2} \mathbf{x}^{T} G(\overline{\boldsymbol{\sigma}}) \mathbf{x}-\mathbf{x}^{T} F(\overline{\boldsymbol{\sigma}})-\overline{\boldsymbol{\sigma}}^{T} \mathbf{d}-a \\
& =\Xi(\mathbf{x}, \overline{\boldsymbol{\sigma}}) .
\end{aligned}
$$

Noting that $P(\overline{\mathbf{x}})=\Xi(\overline{\mathbf{x}}, \overline{\boldsymbol{\sigma}}), \nabla_{\mathbf{x}} \Xi(\overline{\mathbf{x}}, \overline{\boldsymbol{\sigma}})=0$ and $\Xi(\mathbf{x}, \overline{\boldsymbol{\sigma}})$ is a quadratic function with respect to $\mathbf{x}$, we have

$$
\begin{aligned}
P(\mathbf{x})-P(\overline{\mathbf{x}}) & \geq \Xi(\mathbf{x}, \overline{\boldsymbol{\sigma}})-\Xi(\overline{\mathbf{x}}, \overline{\boldsymbol{\sigma}}) \\
& =(\mathbf{x}-\overline{\mathbf{x}}) \nabla_{\mathbf{x}} \Xi(\overline{\mathbf{x}}, \overline{\boldsymbol{\sigma}})+\frac{1}{2}(\mathbf{x}-\overline{\mathbf{x}})^{T} \nabla_{\mathbf{x} \mathbf{x}} \Xi(\overline{\mathbf{x}}, \overline{\boldsymbol{\sigma}})(\mathbf{x}-\overline{\mathbf{x}}) \\
& =\frac{1}{2}(\mathbf{x}-\overline{\mathbf{x}})^{T} G(\overline{\boldsymbol{\sigma}})(\mathbf{x}-\overline{\mathbf{x}}) .
\end{aligned}
$$

If $G(\overline{\boldsymbol{\sigma}}) \succeq 0$, it is easy to find that $\overline{\mathbf{x}}$ is the global minimizer of $(\mathcal{P})$, where, $\overline{\mathbf{x}}$ is contained in the canonical equilibrium equation

$$
G(\overline{\boldsymbol{\sigma}}) \overline{\mathbf{x}}=F(\overline{\boldsymbol{\sigma}}) .
$$

If $G(\overline{\boldsymbol{\sigma}})$ is nonsingular, we have $\overline{\mathbf{x}}=G^{-1}(\overline{\boldsymbol{\sigma}}) F(\overline{\boldsymbol{\sigma}})$.

\section{Implementation techniques}

To solve the optimization problem of $\left(\mathcal{P}^{d}\right)$, we firstly rewrite it to the following form:

$$
\begin{aligned}
\min & \frac{1}{2} t+\boldsymbol{\sigma}^{T} \mathbf{d} \\
\text { subject to : } & t \geq F^{T}(\boldsymbol{\sigma}) G^{-1}(\boldsymbol{\sigma}) F(\boldsymbol{\sigma}) \\
& G(\boldsymbol{\sigma}) \succeq 0 \\
& \boldsymbol{\sigma} \geq 0
\end{aligned}
$$

The global solution of $\left(\mathcal{P}^{d}\right)$ is the same to the problem. Using the Schur complement 4, we can get the equivalent positive(semi) definite condition to (22) and (23)

$$
\left(\begin{array}{cc}
G(\boldsymbol{\sigma}) & F(\boldsymbol{\sigma}) \\
F^{T}(\boldsymbol{\sigma}) & t
\end{array}\right) \succeq 0
$$


and then the optimization problem can be expressed as the standard SDP form

$$
\begin{aligned}
\min & \frac{1}{2} t+\boldsymbol{\sigma}^{T} \mathbf{d} \\
\text { subject to : } & \left(\begin{array}{cc}
G(\boldsymbol{\sigma}) & F(\boldsymbol{\sigma}) \\
F^{T}(\boldsymbol{\sigma}) & t
\end{array}\right) \succeq 0 \\
& \boldsymbol{\sigma} \geq 0
\end{aligned}
$$

If $G(\overline{\boldsymbol{\sigma}}) \succ 0$, we can get the corresponding global solution to $(\mathcal{P})$ by the canonical duality theory. In practice, the estimation of $G(\overline{\boldsymbol{\sigma}})$ may exist little inaccuracy due to the perturbed complementary slackness in primal-dual interior point method and numerical precision. In this study, we use the Cholesky factorization, Condition number and the smallest Eigenvalue of $G(\overline{\boldsymbol{\sigma}})$ to evaluate the positive definiteness comprehensively. If $G(\overline{\boldsymbol{\sigma}})$ is ill conditioned or $\operatorname{det}(G(\overline{\boldsymbol{\sigma}}))=0$, we can add a linear perturbation to the primal objective function and then integrate the canonical dual solutions with the KKT conditions to recover the approximate solution or global solution to primal problem. Details of the techniques are given in the following examples.

\section{Numerical results}

All of the benchmark constrained optimization are from [9], and we keep the number of each problem. In the experiments, we use SeDuMi [11] (a software package which can solve SDP problem) to obtain the canonical dual solutions. The built-in functions fsolve and fminunc in MATLAB are also used to solve the simple nonlinear equations and unconstrained optimization problems.

Example 1: g01

$$
\begin{aligned}
\min f(\mathbf{x})= & 5 \sum_{i=1}^{4} x_{i}-5 \sum_{i=1}^{4} x_{i}^{2}-\sum_{i=5}^{13} x_{i} \\
\text { subject to }: & g_{1}(\mathbf{x})=2 x_{1}+2 x_{2}+x_{10}+x_{11}-10 \leq 0 \\
& g_{2}(\mathbf{x})=2 x_{1}+2 x_{3}+x_{10}+x_{12}-10 \leq 0 \\
& g_{3}(\mathbf{x})=2 x_{2}+2 x_{3}+x_{11}+x_{12}-10 \leq 0 \\
& g_{4}(\mathbf{x})=-8 x_{1}+x_{10} \leq 0 \\
& g_{5}(\mathbf{x})=-8 x_{2}+x_{11} \leq 0 \\
& g_{6}(\mathbf{x})=-8 x_{3}+x_{12} \leq 0 \\
& g_{7}(\mathbf{x})=-2 x_{4}-x_{5}+x_{10} \leq 0 \\
& g_{8}(\mathbf{x})=-2 x_{6}-x_{7}+x_{11} \leq 0 \\
& g_{9}(\mathbf{x})=-2 x_{8}-x_{9}+x_{12} \leq 0
\end{aligned}
$$

where the bounds are $0 \leq x_{i} \leq 1(i=1, \cdots, 9), 0 \leq x_{i} \leq 100(i=10,11,12)$ and $0 \leq x_{13} \leq 1$. 
Solving the canonical dual problem, we can obtain $\overline{\boldsymbol{\sigma}}=$

$\left(\begin{array}{c|c|c|c|c|c|c|c|c|c|c}\sigma_{1} & \sigma_{2} & \sigma_{3} & \sigma_{4} & \sigma_{5} & \sigma_{6} & \sigma_{7} & \sigma_{8} & \sigma_{9} & \sigma_{10} & \sigma_{11} \\ 0.0000 & 0.0000 & 0.0000 & 0.0000 & 0.0000 & 0.0000 & 1.0000 & 1.0000 & 1.0000 & 5.0000 & 5.0000 \\ \sigma_{12} & \sigma_{13} & \sigma_{14} & \sigma_{15} & \sigma_{16} & \sigma_{17} & \sigma_{18} & \sigma_{19} & \sigma_{20} & \sigma_{21} & \sigma_{22} \\ 5.0000 & 7.0001 & 2.0001 & 3.0001 & 2.0001 & 3.0001 & 2.0001 & -0.0000 & -0.0000 & -0.0000 & 1.0001\end{array}\right)$

In this case, $G(\overline{\boldsymbol{\sigma}}) \succeq 0$ but singular, satisfying the global optimality condition. By the KKT condition, we can find that $g_{7}, g_{8}, g_{9}$, bounds of $x_{1}, \cdots, x_{9}$, and $x_{13}$ are active, so we can first get

$$
\left(\begin{array}{c|c|c|c|c|c|c|c|c|c|c|c|c}
x_{1} & x_{2} & x_{3} & x_{4} & x_{5} & x_{6} & x_{7} & x_{8} & x_{9} & x_{10} & x_{11} & x_{12} & x_{13} \\
1 & 1 & 1 & 1 & 1 & 1 & 1 & 1 & 1 & - & - & - & 1
\end{array}\right)
$$

where, " - " means undetermined. Considering that constraints $g_{7}, g_{8}, g_{9}$ are active, solving the corresponding linear equations, we can easily get $x_{10}=$ $3, x_{11}=3, x_{12}=3$. Finally, the global solution to $\mathrm{g} 01$ is $\mathbf{x}^{*}=$

$$
\left(\begin{array}{c|c|c|c|c|c|c|c|c|c|c|c|c}
x_{1} & x_{2} & x_{3} & x_{4} & x_{5} & x_{6} & x_{7} & x_{8} & x_{9} & x_{10} & x_{11} & x_{12} & x_{13} \\
1 & 1 & 1 & 1 & 1 & 1 & 1 & 1 & 1 & 3 & 3 & 3 & 1
\end{array}\right)
$$

and $f\left(\mathbf{x}^{*}\right)=-15$.

Example 2: g04

$$
\min f(\mathbf{x})=5.3578547 x_{3}^{2}+0.8356891 x_{1} x_{5}+37.293239 x_{1}-40792.141
$$

subject to : $g_{1}(\mathbf{x})=85.334407+0.0056858 x_{2} x_{5}+0.0006262 x_{1} x_{4}-0.0022053 x_{3} x_{5}-92 \leq 0$

$$
\begin{aligned}
& g_{2}(\mathbf{x})=-85.334407-0.0056858 x_{2} x_{5}-0.0006262 x_{1} x_{4}+0.0022053 x_{3} x_{5} \leq 0 \\
& g_{3}(\mathbf{x})=80.51249+0.0071317 x_{2} x_{5}+0.0029955 x_{1} x_{2}+0.0021813 x_{3}^{2}-110 \leq 0 \\
& g_{4}(\mathbf{x})=-80.51249-0.0071317 x_{2} x_{5}-0.0029955 x_{1} x_{2}-0.0021813 x_{3}^{2}+90 \leq 0 \\
& g_{5}(\mathbf{x})=9.30096+0.0047026 x_{3} x_{5}+0.0012547 x_{1} x_{3}+0.0019085 x_{3} x_{4}-25 \leq 0 \\
& g_{6}(\mathbf{x})=-9.30096-0.0047026 x_{3} x_{5}-0.0012547 x_{1} x_{3}-0.0019085 x_{3} x_{4}+20 \leq 0
\end{aligned}
$$

where $78 \leq x_{1} \leq 102,33 \leq x_{2} \leq 45$ and $27 \leq x_{i} \leq 45(i=3,4,5)$.

Solving the canonical dual problem, we can obtain $\overline{\boldsymbol{\sigma}}=$

$$
\left(\begin{array}{c|c|c|c|c|c|c|c|c|c|c}
\sigma_{1} & \sigma_{2} & \sigma_{3} & \sigma_{4} & \sigma_{5} & \sigma_{6} & \sigma_{7} & \sigma_{8} & \sigma_{9} & \sigma_{10} & \sigma_{11} \\
336.8388 & 0.0000 & 0.0001 & 0.0002 & 0.0003 & 798.2826 & 2.0310 & 6.1233 & 0.0001 & 1.6054 & 1.1849
\end{array}\right)
$$

In this case, $G(\overline{\boldsymbol{\sigma}}) \succ 0$ and $\operatorname{cond}(G(\overline{\boldsymbol{\sigma}}))=9.7330 e 5$, satisfying the global optimality condition, so we can get $\overline{\mathbf{x}}=$

$$
\left(\begin{array}{c|c|c|c|c}
x_{1} & x_{2} & x_{3} & x_{4} & x_{5} \\
77.9452 & 33.0179 & 29.7345 & 44.9884 & 38.2523
\end{array}\right)
$$

Noting that the condition number is large, according to the KKT condition, we can first get

$$
\left(\begin{array}{l|l|l|l|l}
x_{1} & x_{2} & x_{3} & x_{4} & x_{5} \\
78 & 33 & - & 45 & -
\end{array}\right)
$$


Considering that constraints $g_{1}, g_{6}$ are active, solving the corresponding linear equations, we can easily get $x_{3}=29.995256025681599, x_{5}=36.775812905788207$. Finally, the global solution to g04 is $\mathbf{x}^{*}=$

$$
\left(\begin{array}{c|c|c|c|c}
x_{1} & x_{2} & x_{3} & x_{4} & x_{5} \\
78 & 33 & 29.995256025681599 & 45 & 36.775812905788207
\end{array}\right)
$$

and $f\left(\mathbf{x}^{*}\right)=-3.0666 e 4$.

Remark 1 We use the inverse of $G(\overline{\boldsymbol{\sigma}})$ because only its smallest eigenvalues approximates to zero although its condition number is large. As a matter of fact, the solution $\overline{\mathbf{x}}$ causes only little infeasibility of the first constraint. By integrating the canonical dual solutions and the KKT conditions, we claim that $x_{1}, x_{2}$ and $x_{4}$ are determined in the first stage.

Example 3: g07

$$
\begin{aligned}
\min f(\mathbf{x})=x_{1}^{2} & +x_{2}^{2}+x_{1} x_{2}-14 x_{1}-16 x_{2}+\left(x_{3}-10\right)^{2}+4\left(x_{4}-5\right)^{2}+\left(x_{5}-3\right)^{2} \\
& +2\left(x_{6}-1\right)^{2}+5 x_{7}^{2}+7\left(x_{8}-11\right)^{2}+2\left(x_{9}-10\right)^{2}+\left(x_{10}-7\right)^{2}+45
\end{aligned}
$$

subject to : $g_{1}(\mathbf{x})=-105+4 x_{1}+5 x_{2}-3 x_{7}+9 x_{8} \leq 0$

$$
\begin{aligned}
& g_{2}(\mathbf{x})=10 x_{1}-8 x_{2}-17 x_{7}+2 x_{8} \leq 0 \\
& g_{3}(\mathbf{x})=-8 x_{1}+2 x_{2}+5 x_{9}-2 x_{10}-12 \leq 0 \\
& g_{4}(\mathbf{x})=3\left(x_{1}-2\right)^{2}+4\left(x_{2}-3\right)^{2}+2 x_{3}^{2}-7 x_{4}-120 \leq 0 \\
& g_{5}(\mathbf{x})=5 x_{1}^{2}+8 x_{2}+\left(x_{3}-6\right)^{2}-2 x_{4}-40 \leq 0 \\
& g_{6}(\mathbf{x})=x_{1}^{2}+2\left(x_{2}-2\right)^{2}-2 x_{1} x_{2}+14 x_{5}-6 x_{6} \leq 0 \\
& g_{7}(\mathbf{x})=0.5\left(x_{1}-8\right)^{2}+2\left(x_{2}-4\right)^{2}+3 x_{5}^{2}-x_{6}-30 \leq 0 \\
& g_{8}(\mathbf{x})=-3 x_{1}+6 x_{2}+12\left(x_{9}-8\right)^{2}-7 x_{10} \leq 0
\end{aligned}
$$

where $-10 \leq x_{i} \leq 10(i=1, \cdots, 10)$.

Solving the canonical dual problem, we can obtain $\overline{\boldsymbol{\sigma}}=$

$$
\left(\begin{array}{c|c|c|c|c|c|c|c|c}
\sigma_{1} & \sigma_{2} & \sigma_{3} & \sigma_{4} & \sigma_{5} & \sigma_{6} & \sigma_{7} & \sigma_{8} & \sigma_{9} \\
1.7168 & 0.4746 & 1.3760 & 0.0205 & 0.3120 & 0.2871 & 0.0000 & 0.0000 & 0.0000 \\
\sigma_{10} & \sigma_{11} & \sigma_{12} & \sigma_{13} & \sigma_{14} & \sigma_{15} & \sigma_{16} & \sigma_{17} & \sigma_{18} \\
0.0000 & 0.0000 & 0.0000 & 0.0000 & 0.0000 & 0.0000 & 0.0000 & 0.0000 & 0.0000
\end{array}\right)
$$

In this case, $G(\overline{\boldsymbol{\sigma}}) \succ 0$ and $\operatorname{cond}(G(\overline{\boldsymbol{\sigma}}))=7.0000$, satisfying the global optimality condition, so we can get $\mathbf{x}^{*}=$

$$
\left(\begin{array}{c|c|c|c|c|c|c|c|c|c}
x_{1} & x_{2} & x_{3} & x_{4} & x_{5} & x_{6} & x_{7} & x_{8} & x_{9} & x_{10} \\
2.1721 & 2.3636 & 8.7746 & 5.0959 & 0.9903 & 1.4307 & 1.3218 & 9.8286 & 8.2800 & 8.3760
\end{array}\right)
$$

and $f\left(\mathbf{x}^{*}\right)=24.3111$. Note that there exists little infeasibility due to numerical precision. 
Example 4: g10

$$
\begin{aligned}
& \min f(\mathbf{x})=x_{1}+x_{2}+x_{3} \\
& \text { subject to : } g_{1}(\mathbf{x})=-1+0.0025\left(x_{4}+x_{6}\right) \leq 0 \\
& g_{2}(\mathbf{x})=-1+0.0025\left(x_{5}+x_{7}-x_{4}\right) \leq 0 \\
& g_{3}(\mathbf{x})=-1+0.01\left(x_{8}-x_{5}\right) \leq 0 \\
& g_{4}(\mathbf{x})=-x_{1} x_{6}+833.33252 x_{4}+100 x_{1}-83333.333 \leq 0 \\
& g_{5}(\mathbf{x})=-x_{2} x_{7}+1250 x_{5}+x_{2} x_{4}-1250 x_{4} \leq 0 \\
& g_{6}(\mathbf{x})=-x_{3} x_{8}+1250000+x_{3} x_{5}-2500 x_{5} \leq 0
\end{aligned}
$$

where $100 \leq x_{1} \leq 10000,1000 \leq x_{i} \leq 10000(i=2,3)$ and $10 \leq x_{i} \leq 1000(i=$ $4, \cdots, 8)$

Solving the canonical dual problem, we can obtain $\overline{\boldsymbol{\sigma}}=$

$$
\left(\begin{array}{c|c|c|c|c|c|c}
\sigma_{1} & \sigma_{2} & \sigma_{3} & \sigma_{4} & \sigma_{5} & \sigma_{6} & \sigma_{7} \\
9.2834 & 28.9205 & 5.8893 & 0.0001 & 0.0001 & 0.0001 & 0.0001 \\
\sigma_{8} & \sigma_{9} & \sigma_{10} & \sigma_{11} & \sigma_{12} & \sigma_{13} & \sigma_{14} \\
0.0001 & 0.0001 & 0.0000 & 0.0000 & 0.0000 & 0.0000 & 0.0000
\end{array}\right)
$$

In this case, $G(\overline{\boldsymbol{\sigma}}) \succ 0$ and $\operatorname{cond}(G(\overline{\boldsymbol{\sigma}}))=749.4514$, satisfying the global optimality condition. However, the $\max (\operatorname{eig}(G(\overline{\boldsymbol{\sigma}})))=2.5743 e-4$, which is too small, so we cannot use the inverse of $G(\overline{\boldsymbol{\sigma}})$ directly. By the KKT condition, we can find that constraints $g_{1}, g_{2}, g_{3}$ are active, and all of the box constraints are inactive. That is to say, the problem is equivalent to a linear programming problem with linear constraints, which indicates that $g_{4}, g_{5}, g_{6}$ must be active. Fixing $x_{4}, x_{5}$, we have

$$
\left\{\begin{array}{l}
x_{1}=\frac{83333.333-833.33252 x_{4}}{x_{4}-300} \\
x_{2}=\frac{1250 x_{4}-1250 x_{5}}{x_{5}-400} \\
x_{3}=12500-25 x_{5} \\
x_{6}=400-x_{4} \\
x_{7}=400+x_{4}-x_{5} \\
x_{8}=100+x_{5}
\end{array}\right.
$$

As a result, we can reduce the problem to

$$
\min f(\mathbf{x})=\frac{83333.333-833.33252 x_{4}}{x_{4}-300}+\frac{1250 x_{4}-1250 x_{5}}{x_{5}-400}+12500-25 x_{5}
$$

Taking the box constraints of $x_{1}, \cdots, x_{8}$ into consideration, when using $(100,200)$ as an initial point for the unconstrained optimization problem with two variables, it is easy to get the only minimum $x_{4}=182.0176995811199$ and $x_{5}=$ 295.6011732779338. Utilizing the equations obtained by the complementary slackness, finally, we have $x_{1}=579.3066844253549 x_{2}=1359.970668051655$, 
$x_{3}=5109.970668051655, x_{6}=217.9823004188801, x_{7}=286.4165263031861$, $x_{8}=395.6011732779338$ and $f\left(\mathbf{x}^{*}\right)=7049.248020528666$.

Remark 2 We don't use the inverse of $G(\overline{\boldsymbol{\sigma}})$ directly because all of its eigenvalues are approximately zeros. And the reason why we still use the canonical dual solutions as useful heuristics is that the $G(\overline{\boldsymbol{\sigma}})$ is slightly positive definite due to the perturbed complementary slackness caused by the SeDuMi. Since all of the box constraints are inactive and the objective function is linear, it is not difficult to imagine that all of the constraints must be active. Note that the constraints of $x_{4}$ and $x_{5}$ are changed when solving the unconstrained optimization problem since constraints of $x_{1}, x_{2}, x_{3}$ and $x_{6}, x_{7}, x_{8}$ must be satisfied.

Example 5: g18

$$
\begin{aligned}
& \min f(\mathbf{x})=-0.5\left(x_{1} x_{4}-x_{2} x_{3}+x_{3} x_{9}-x_{5} x_{9}+x_{5} x_{8}-x_{6} x_{7}\right) \\
& \text { subject to : } g_{1}(\mathbf{x})=x_{3}^{2}+x_{4}^{2}-1 \leq 0 \\
& g_{2}(\mathbf{x})=x_{9}^{2}-1 \leq 0 \\
& g_{3}(\mathbf{x})=x_{5}^{2}+x_{6}^{2}-1 \leq 0 \\
& g_{4}(\mathbf{x})=x_{1}^{2}+\left(x_{2}-x_{9}\right)^{2}-1 \leq 0 \\
& g_{5}(\mathbf{x})=\left(x_{1}-x_{5}\right)^{2}+\left(x_{2}-x_{6}\right)^{2}-1 \leq 0 \\
& g_{6}(\mathbf{x})=\left(x_{1}-x_{7}\right)^{2}+\left(x_{2}-x_{8}\right)^{2}-1 \leq 0 \\
& g_{7}(\mathbf{x})=\left(x_{3}-x_{5}\right)^{2}+\left(x_{4}-x_{6}\right)^{2}-1 \leq 0 \\
& g_{8}(\mathbf{x})=\left(x_{3}-x_{7}\right)^{2}+\left(x_{4}-x_{8}\right)^{2}-1 \leq 0 \\
& g_{9}(\mathbf{x})=x_{7}^{2}+\left(x_{8}-x_{9}\right)^{2}-1 \leq 0 \\
& g_{10}(\mathbf{x})=x_{2} x_{3}-x_{1} x_{4} \leq 0 \\
& g_{11}(\mathbf{x})=-x_{3} x_{9} \leq 0 \\
& g_{12}(\mathbf{x})=x_{5} x_{9} \leq 0 \\
& g_{13}(\mathbf{x})=x_{6} x_{7}-x_{5} x_{8} \leq 0
\end{aligned}
$$

where $-10 \leq x_{1} \leq 10,(i=1, \cdots, 8)$ and $0 \leq x_{9} \leq 20$.

Solving the canonical dual problem, we can obtain $\overline{\boldsymbol{\sigma}}=$

$\left(\begin{array}{c|c|c|c|c|c|c|c|c|c|c}\sigma_{1} & \sigma_{2} & \sigma_{3} & \sigma_{4} & \sigma_{5} & \sigma_{6} & \sigma_{7} & \sigma_{8} & \sigma_{9} & \sigma_{10} & \sigma_{11} \\ 0.1444 & 0.0000 & 0.1444 & 0.1445 & 0.0000 & 0.1442 & 0.1441 & 0.0000 & 0.1445 & 0.0000 & 0.0000 \\ \sigma_{12} & \sigma_{13} & \sigma_{14} & \sigma_{15} & \sigma_{16} & \sigma_{17} & \sigma_{18} & \sigma_{19} & \sigma_{20} & \sigma_{21} & \sigma_{22} \\ 0.0000 & 0.0000 & -0.0000 & -0.0000 & -0.0000 & -0.0000 & -0.0000 & -0.0000 & -0.0000 & -0.0000 & 0.0000\end{array}\right)$

In this case, $G(\overline{\boldsymbol{\sigma}}) \succ 0$ and $\operatorname{cond}(G(\overline{\boldsymbol{\sigma}}))=7.1887 e 7$, satisfying the global optimality condition. However, the condition number is large. Taking the KKT conditions into account, we can conclude that constraints $g_{1}, g_{3}, g_{4}, g_{6}, g_{7}$, $g_{9}$ are active since the corresponding $\sigma_{1}, \sigma_{3}, \sigma_{4}, \sigma_{6}, \sigma_{7}, \sigma_{9}$ are not zeros. But it becomes still difficult to solve the nonlinear equations. Considering that several eigenvalues of $G(\overline{\boldsymbol{\sigma}})$ are zeros and there exists no linear term in the objective function, and in this situation, we add a small linear perturbation 
$0.05\left(x_{1}+\cdots, x_{9}\right)$ to the primal objective function. Solving the perturbed canonical dual problem, we get $G(\overline{\boldsymbol{\sigma}}) \succ 0$ and $\operatorname{cond}(G(\overline{\boldsymbol{\sigma}}))=1.4592 e 3$ and the smallest eigenvalue of $G(\overline{\boldsymbol{\sigma}})$ is 0.0021 . Therefore, we can get $\overline{\mathbf{x}}=$

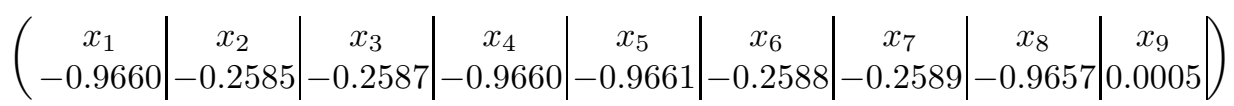

and $f(\overline{\mathbf{x}})=-0.8663$. Note that there exists little infeasibility due to numerical precision.

Remark 3 The solution we get is quite different from the best known solution. According to the canonical duality theory, the global solution to this problem is not unique. The linear perturbation technique can only help to find one of the global solutions. As a matter of fact, the following solutions $\overline{\mathbf{x}}=$

$$
\begin{aligned}
& \left(\begin{array}{c|c|c|c|c|c|c|c|c}
x_{1} & x_{2} & x_{3} & x_{4} & x_{5} & x_{6} & x_{7} & x_{8} & x_{9} \\
0.0450 & -0.0387 & 0.8663 & -0.4999 & 0.0004 & -1.0001 & 0.8878 & 0.5000 & 0.9604
\end{array}\right), \\
& \left(\begin{array}{c|c|c|c|c|c|c|c|c}
x_{1} & x_{2} & x_{3} & x_{4} & x_{5} & x_{6} & x_{7} & x_{8} & x_{9} \\
0.0689 & -0.9972 & 0.9088 & -0.4179 & 0.0920 & -0.9959 & 0.8986 & -0.4388 & 0.0009
\end{array}\right),
\end{aligned}
$$

and

$$
\left(\begin{array}{c|c|c|c|c|c|c|c|c}
x_{1} & x_{2} & x_{3} & x_{4} & x_{5} & x_{6} & x_{7} & x_{8} & x_{9} \\
0.6888 & -0.7257 & 0.9693 & 0.2454 & 0.6973 & -0.7173 & 0.9726 & 0.2332 & -0.0006
\end{array}\right)
$$

can all be considered as approximate solutions, which are obtained by the proposed techniques.

\section{Conclusion}

We have applied the canonical duality theory to solve a class of CEC benchmark constrained optimization problems. Experimental results show that some of the examples can be solved directly, some of them can be solved by integrating the canonical dual solutions and KKT conditions, and some can be solved approximately by adding a small linear perturbation term.

\section{References}

1. Bazaraa, M.S., Sherali, H.D., Shetty, C.M.: Nonlinear programmming: Theory and Algorithms, 3rd, edn. Wiley, New York (2006)

2. Boyd S., Vandenberghe L.: Convex optimization, Cambridge University Press (2004)

3. Coello, C.A.C.: Theoretical and numerical constraint-handling techniques used with evolutionary algorithms: a survey of the state of the art. Comput. Methods Appl. Mech. Engrg. 191, 1245-1287 (2002)

4. Cottle, R.W.: Manifestations of Schur complement. Linear algebra and its applications. 8, 189-211 (1974) 
5. Fang, S.C., Gao, D.Y., Sheu R.L. and Wu, S.Y.: Canonical dual approach for solving 0-1 quadratic programming problems. J. Ind. and Manag. Optim. 4, 125-142 (2008)

6. Gao, D.Y., Ruan N., Sherali H.D.: Solutions and optimality criteria for nonconvex constrained global optimization problems with connections between canonical and Lagrandian duality. J. Glob. Optim. 45, 473-497 (2009)

7. Gao, D.Y.: Caonical duality theory: Unified understanding and generalized solution for global optimization problems. Computers and Chemical Engineering. 33, 1964-1972 (2009)

8. Gao, D.Y., Ruan N.: Solutions to quadratic minimization problems with box and integer constraints. J. Glob. Optim. 47, 463-484 (2010)

9. Liang, J.J., Runarsson, T.P., Mezura-Montes, E., Clerc, M., Suganthan, P.N., Coello, C.A.C. and Deb, K.: Problem definitions and evaluation criteria for the CEC 2006 (special session on constrained real-parameter optimization). Nanyang Technol. Univ., Singapore, Tech. Rep. (2006)

10. Michalewicz Z.: A survey of constraint handling techniques in evolutionary computation methods. in: J.R. McDonnell, R.G. Reynolds, D.B. Fogel (Eds.), Proceedings of the Fourth Annual Conference on Evolutionary Programming, MIT Press, Cambridge, MA, pp. 135$155(1995)$

11. Sturn, J.F.: Using SeDuMi 1.02, a MATLAB toolbox for optimization over symmetric cones. Optim. Meth. Softw. 11, 625-653 (1999)

12. Wang, Z.B., Fang, S.C., Gao, D.Y., Xing, W.X.: Canonical dual approach to solving the maximum cut problem. Journal of Global Optimization, 54, 341-352 (2012) 